\title{
Perfil de sustentabilidad tridimensional en el valle agrícola del municipio de Casimiro Castillo, Jalisco, México
}

\author{
Perfil de sustentabilidad tridimensional en el valle agrícola del municipio de \\ Casimiro Castillo, Jalisco, México
}

\author{
Fallad Jalil ${ }^{1}$, Gómez José Eduardo ${ }^{1}$, Hueso Eva Judith ${ }^{1}$, Castañeda Alfredo ${ }^{1}$, \\ Teodoro Ramón ${ }^{1}$, Silva Rafaela Blanca ${ }^{1}$
}

\begin{abstract}
RESUMEN
Durante muchos años se ha venido generando una gran conciencia sobre los estudios de impacto ambiental, económico y social para encontrar características clave sobre el estado de las actividades productivas, incluyendo a la agricultura. Esto con la finalidad de detectar problemas y que, posteriormente, los gobiernos puedan crear programas de desarrollo para promover la sostenibilidad en todo el mundo. Las nuevas exigencias han conducido a una necesidad imperiosa de hacer un cambio efectivo hacia actividades agrícolas, como riqueza social pero bajo el enfoque de sostenibilidad. La metodología del IRS utilizada en esta investigación permitió un estudio especializado de cuestiones ecológicas, sociales y económicas. Los resultados sugieren que la sostenibilidad del valle del municipio Casimiro Castillo ubicado en el estado de Jalisco, México, es muy baja, en comparacióncon otros municipios cercanos.

Palabras clave: IRS, sustentabilidad, Casimiro Castillo, Jalisco.
\end{abstract}

\section{ABSTRACT}

For many years, high awareness about environmental, economic, and social impact studies have been made to find key features about the status of productive activities, including agriculture, to detect problems. Then, the government can create developing programs to promote sustainability worldwide. This need led to a compelling need to make an effective shift towards agricultural activities, and social wealth under the sustainability approach. The SRI Methodology used in this research allowed a specialized study of ecological, social, and economic issues. The results of this study suggested that the sustainability of the Casimiro Castillo Valley located in the state of Jalisco, Mexico, is very low; there is a difference towards other municipalities nearby.

Keywords: IRS, Sustainable, Casimiro Castillo, Jalisco.

\section{Introducción}

El desarrollo sostenible, como modelo en construcción, considera el trabajo, la innovación, la construcción e invención, y le sirve a la sociedad para superar los viejos y actuales problemas de la humanidad. Por lo tanto, se trata de una manera diferente de pensar, hacer y vivir basándose en las necesidades de sus propios actores, y no es como algunos autores señalan un problema de crecimiento continuo, intentando confundir a sus detractores (WECD, 1987; Brown, et al., 1987; Bowers, 1997).
El estudio tuvo como objetivo la evaluación de la sustentabilidad del valle agrícola del municipio de Casimiro Castillo, Jalisco, México, mediante la aplicación de la metodología del Índice de Sustentabilidad Relativa (IRS) diseñado por Casas et al., 2009. Este instrumento permite cuantificar la sustentabilidad y la identificación de los elementos clave que pueden servir para el diseño de las estrategias encaminadas al desarrollo agrícola local, regional y nacional.

El ISR es una metodología ad hoc para evaluar la sostenibilidad a nivel comunitario o de agroecosistemas propuesta por Casas et al. (2001), que en este ensayo

\footnotetext{
1 University of Guadalajara. Guadalajara, México.

* Autor por correspondencia: jfallad@cucsur.udg.mx
} 
se describe y complementa para su aplicación. El Índice parte del concepto de sostenibilidad.

\section{Metodología}

La evaluación de sustentabilidad del valle agrícola del municipio de Casimiro Castillo, Jalisco, México, se obtuvo a partir de un diagnóstico con la aplicación de la metodología del IRS. Esta metodología integró tres variables de diferente naturaleza (social, económica, ambiental) y las unidades en un producto adimensional. Para ello, se llevó a cabo un proceso de estandarización de variables ambientales como agua, suelo, biodiversidad, manejo y área de cultivo (temporal, riego, fertilizantes químicos u orgánicos y niveles de productividad); variables sociales: características de la población humana, su densidad, el empleo, la salud, la alimentación, la educación, la migración, participación social, política e instrumentos de trabajo agrícola; y variables económicas como los bienes e instrumentos tecnológicos y de capital para llevar a cabo sus actividades y el equilibrio de sus actividades económicas (ingresos-egresos). Esta metodología no tuvo como objetivo lograr un estudio especializado de temas ecológicos, sociales y económicos sino determinar un índice de sustentabilidad que se encuentra ligado a la productividad agrícola de los productores de caña de azúcar del municipio de Casimiro Castillo. Los temas, así como las variables seleccionadas, fueron aquellos en los que hay más o menos coincidencia para la evaluación de la sustentabilidad. Las variables que se escogieron para estimar la sustentabilidad no son las únicas o restrictivas y podrían ser susceptibles de modificarse para cumplir con las necesidades de los objetivos de otros estudios (Tablas 2, 3 y 4).
El diagnóstico, como ya se ha mencionado, se realizó con la aplicación del IRS para cuantificar la sostenibilidad en función de las dimensiones ambientales, sociales y económicas de la región de Casimiro Castillo. La evaluación de sostenibilidad consideró la información de las variables sociales, económicas y ambientales en las comunidades ubicadas en el valle agrícola de este municipio.

\section{Tipo y tamaño de muestra}

El tipo de muestreo resultó conveniente ya que no fue posible acceder a todos los agricultores del municipio para un estudio más extenso y sólo se pudo entrevistar a algunos de ellos.

En consecuencia, el tamaño de la muestra se determinó mediante los criterios de saturación y/o redundancia.

\section{Lugar del estudio}

La investigación se llevó a cabo en el valle agrícola del municipio de Casimiro Castillo, ubicado en la costa sur del estado de Jalisco, México (Figura 1).

El municipio de Casimiro Castillo está situado al suroeste del estado de Jalisco, en las coordenadas extremas de $19^{\circ} 21^{\prime} 40^{\prime \prime}$ a $19^{\circ} 45^{\prime} 00^{\prime \prime}$ de latitud norte y $104^{\circ} 18^{\prime} 00^{\prime \prime}$ a $104^{\circ} 31^{\prime} 32^{\prime \prime}$ de longitud oeste, a una altura de 366 metros sobre el nivel del mar.

Limita al norte con los municipios de Villa Purificación y Autlán de Navarro; al este con los municipios de Autlán de Navarro y Cuautitlán de García Barragán; al sur con los municipios de Cuautitlán de García Barragán y La Huerta; y al oeste con los municipios de La Huerta y Villa Purificación.

Tabla 1. Evaluación de la sostenibilidad y la contribución de las dimensiones para el desarrollo sustentable del Valle Agrícola en el municipio de Casimiro Castillo, Jalisco, México, con base en datos estadísticos.

\begin{tabular}{lccccccc}
\hline \multicolumn{1}{c}{ Municipio } & $\begin{array}{c}\text { Dimensión } \\
\text { Económica }\end{array}$ & $\begin{array}{c}\text { Dimensión } \\
\text { Social }\end{array}$ & $\begin{array}{c}\text { Dimensión } \\
\text { Ambiental }\end{array}$ & IRS & $\begin{array}{c}\text { \% Contribución } \\
\text { a sustentabilidad }\end{array}$ & $\begin{array}{c}\text { Calificación de } \\
\text { sustentabilidad }\end{array}$ & $\begin{array}{c}\text { Prioridad } \\
\text { de atención }\end{array}$ \\
\hline Casimiro Castillo & 0,414 & 0,421 & 0,469 & 0,418 & 6,74 & $\begin{array}{c}\text { sustentabilidad } \\
\text { muy baja }\end{array}$ & 7 \\
\hline $\begin{array}{l}\text { Contribución a la } \\
\text { Dimensión dimension }\end{array}$ & 0,491 & 0,482 & 0,477 & 0,442 & 9,74 & $\begin{array}{c}\text { sustentabilidad } \\
\text { muy baja }\end{array}$ & 7 \\
\hline $\begin{array}{l}\text { Calificación por } \\
\text { Dimensión }\end{array}$ & $\begin{array}{c}\text { Muy Baja } \\
\text { Contribución }\end{array}$ & $\begin{array}{c}\text { Muy Baja } \\
\text { Contribución }\end{array}$ & $\begin{array}{c}\text { Muy Baja, } \\
\text { ausente }\end{array}$ & & & & \\
\hline
\end{tabular}


Tabla 2. Listado de variables sociales.

\begin{tabular}{cllll}
\hline Listado & \multicolumn{1}{c}{ Variable } & \multicolumn{1}{c}{ Definición } & \multicolumn{1}{c}{ Tendencia R.S. } & Unidades \\
\hline Y2 & Edad & Años de edad que el agricultor tiene actualmente. & $\begin{array}{l}\text { Entre más joven o maduro } \\
\text { mayor TRS }\end{array}$ & Número de años \\
\hline Y3 & $\begin{array}{l}\text { Integrantes de la } \\
\text { familia }\end{array}$ & $\begin{array}{l}\text { Total de miembros que integran el núcleo } \\
\text { familiar. }\end{array}$ & $\begin{array}{l}\text { Menor número de miembros de } \\
\text { familia mayor TRS }\end{array}$ & Número de personas \\
\hline Y4 & Grado escolar & Número de años de estudio que tiene la familia. & A mayor gasto mayor TRS & Número de años \\
\hline Y5 & $\begin{array}{l}\text { Equidad de género } \\
\text { en la educación }\end{array}$ & $\begin{array}{l}\text { Diferencia de años de estudio oficiales entre } \\
\text { hombres y mujeres }\end{array}$ & $\begin{array}{l}\text { Mayor tiempo dedicado a su } \\
\text { tierra mayor TRS }\end{array}$ & Número de años de \\
Y6 & Íncolaridad oficial
\end{tabular}

Tabla 3. Listado de variables económicas.

\begin{tabular}{|c|c|c|c|c|}
\hline Listado & Variable & Definición & $\begin{array}{l}\text { Tendencia Relativa de } \\
\text { Sustentabilidad (TRS). }\end{array}$ & Unidades \\
\hline $\mathrm{Z} 2$ & $\begin{array}{l}\text { Ingreso externo } \\
\text { anual }\end{array}$ & $\begin{array}{l}\text { Ingreso per cápita anual que percibe cada } \\
\text { miembro de la familia que trabaja en una } \\
\text { actividad diferente externa al trabajo propio } \\
\text { del campo. }\end{array}$ & $\begin{array}{l}\text { A menor ingreso per cápita } \\
\text { externo mayor TRS }\end{array}$ & $\$$ \\
\hline $\mathrm{Z} 4$ & Gasto per cápita & $\begin{array}{l}\text { Gasto total que destina el agricultor en } \\
\text { alimentos, educación, salud y vestido por } \\
\text { miembro de la familia. }\end{array}$ & A mayor gasto mayor TRS & $\$$ \\
\hline $\mathrm{Z5}$ & $\begin{array}{l}\text { Tiempo dedicado } \\
\text { al campo }\end{array}$ & $\begin{array}{l}\text { Meses que el agricultor destina a trabajar } \\
\text { su tierra. }\end{array}$ & $\begin{array}{l}\text { Mayor tiempo dedicado a su } \\
\text { tierra mayor TRS }\end{array}$ & Meses del año \\
\hline Z6 & Trabajo externo & $\begin{array}{l}\text { Número de jornales } \mathrm{x} \text { ha que contrata el } \\
\text { agricultor como mano de obra externa. }\end{array}$ & $\begin{array}{l}\text { A menor número de jornales } \\
\text { contratados mayor TRS }\end{array}$ & Número de personas \\
\hline $\mathrm{Z7}$ & Utilidad agrícola & $\begin{array}{l}\text { Utilidad per cápita anual por la venta de la } \\
\text { producción de sus cultivos. }\end{array}$ & A mayor utilidad mayor TRS & $\$$ \\
\hline $\mathrm{Z} 17$ & $\begin{array}{l}\text { Saldo per cápita } \\
\text { anual }\end{array}$ & $\begin{array}{l}\text { Es el resultado del ingreso anual per cápita } \\
\text { por mano de obra externa, más ingreso anual } \\
\text { por renta de su tierra, más la utilidad por la } \\
\text { producción de sus cultivos más el ingreso } \\
\text { por remesas, MENOS el gasto realizado por } \\
\text { alimentación, educación, salud y vestido más } \\
\text { los costos de producción. }\end{array}$ & A mayor saldo mayor TRS & $\$$ \\
\hline $\mathrm{Z} 21$ & $\begin{array}{l}\text { Limitaciones de la } \\
\text { comercialización }\end{array}$ & $\begin{array}{l}\text { Factores que limitan la comercialización de } \\
\text { los productos agrícolas. }\end{array}$ & $\begin{array}{l}\text { A menos factores limitantes } \\
\text { mayor TRS }\end{array}$ & $\begin{aligned} 1= & \text { Ninguno } \\
2= & \text { Calidad de sus productos } \\
3= & \text { La falta de liquidez para } \\
& \text { pagar deudas } \\
4= & \text { Altos costos de producción } \\
5= & \text { La falta de organización }\end{aligned}$ \\
\hline
\end{tabular}


Tabla 4. Listado de variables ambientales.

\begin{tabular}{|c|c|c|c|c|}
\hline Listado & Variable & Definición & Tendencia R.S. & Unidades \\
\hline $\mathrm{X} 1$ & Superficie per cápita & $\begin{array}{l}\text { Total de superficie agrícola que le } \\
\text { corresponde a cada miembro de la } \\
\text { familia }\end{array}$ & Mayor superficie per cápita mayor TRS & $\mathrm{Ha}$ \\
\hline $\mathrm{X} 3$ & Productividad media & $\begin{array}{l}\text { Se refiere a la productividad media } \\
\text { anual obtenida de toneladas por hectárea } \\
\text { 2007-2009 }\end{array}$ & $\begin{array}{l}\text { Mayor número de toneladas obtenidas } \\
\text { de producción por ha mayor TRS }\end{array}$ & $\mathrm{T} /$ ha/año \\
\hline $\mathrm{X} 4$ & Estabilidad en la producción & Rango de productividad 2007-2009 & $\begin{array}{l}\text { A menor valor de la diferencia del } \\
\text { rango mayor TRS }\end{array}$ & T. $x$ ha \\
\hline $\mathrm{X} 5$ & Basura & $\begin{array}{l}\text { Kilogramos de basura per cápita que } \\
\text { desecha en su hogar diariamente }\end{array}$ & $\begin{array}{l}\text { Entre menos basura deseche mayor } \\
\text { TRS }\end{array}$ & Kilogramos \\
\hline $\mathrm{X} 6$ & Energía eléctrica & $\begin{array}{l}\text { Gasto per cápita anual de energía } \\
\text { eléctrica }\end{array}$ & Entre menor gasto mayor TRS & Kwatts \\
\hline $\mathrm{X} 7$ & Superficie de riego & $\begin{array}{l}\text { Superficie de riego destinada para la } \\
\text { agricultura }\end{array}$ & $\begin{array}{l}\text { A mayor superficie más cultivos } \\
\text { sembrados en el año por tanto mayor } \\
\text { TRS }\end{array}$ & $\mathrm{Ha}$ \\
\hline $\mathrm{X} 8$ & Superficie de temporal & $\begin{array}{l}\text { Superficie agrícola destinada para la } \\
\text { agricultura }\end{array}$ & A mayor superficie mayor TRS & $\mathrm{Ha}$ \\
\hline $\mathrm{X} 9$ & Superficie sin sembrar & $\begin{array}{l}\text { Superficie promedio que dejo sin } \\
\text { sembrar del 2007-2009 }\end{array}$ & Entre menos deje mayor TRS & ha/año \\
\hline $\mathrm{X} 14$ & $\begin{array}{l}\text { Proporción de superficie } \\
\text { sembrada }\end{array}$ & $\begin{array}{l}\text { Proporción de superficie sembrada } \\
\text { del total }\end{array}$ & $\begin{array}{l}\text { Mayor proporción de superficie } \\
\text { sembrada mayor TRS }\end{array}$ & $\mathrm{Ha}$ \\
\hline $\mathrm{X} 18$ & Caña & $\begin{array}{l}\text { Superficie total sembrada de caña en } \\
\text { el } 2007,2008 \text { y } 2009\end{array}$ & $\begin{array}{l}\text { Mayor número de cultivos sembrados y } \\
\text { mayor superficie sembrada mayor TRS }\end{array}$ & $\mathrm{Ha}$ \\
\hline $\mathrm{X} 18.1$ & Maíz & $\begin{array}{l}\text { Superficie total sembrada de maíz de } \\
\text { temporal en el } 2007,2008 \text { y } 2010\end{array}$ & $\begin{array}{l}\text { Mayor número de cultivos sembrados y } \\
\text { mayor superficie sembrada mayor TRS }\end{array}$ & $\mathrm{Ha}$ \\
\hline X 21.1 & Tipo de abono empleado & $\begin{array}{l}\text { empleo de abono orgánico y bio- } \\
\text { insecticidas; líquido (lixiviados). Sólido } \\
\text { (Composta o estiércol de ganado) }\end{array}$ & $\begin{array}{l}\text { A mayor uso de productos orgánicos } \\
\text { mayor TRS }\end{array}$ & $\begin{array}{l}\text { 1= Composta } \\
2=\text { Estiércol de ganado } \\
3=\text { lixiviados de lombriz }\end{array}$ \\
\hline
\end{tabular}

\section{Criterios de selección comunitaria}

La técnica de la encuesta consistió en la aplicación sistemática de un cuestionario a las personas que viven en la mencionada comunidad. Los sujetos encuestados son de ambos sexos y adultos, y puedensaben leer y escribir.

Al comienzo se trató de incluir a todos los productores de caña de azúcar del valle agrícola del municipio, pero no hubo mucho interés por parte de los agricultores en áreas más remotas. Por ello el estudio se centró en aquellas zonas donde los agricultores decidieron participar voluntariamente.

Debido a las características de este trabajo, se utilizaron técnicas de investigación social, económica y ambiental que permitieron aplicar algunas reglas y operaciones para el uso del instrumento. La técnica de encuesta consistió en la interrogación sistemática de individuos para conocer el funcionamiento de la lógica económica, ambiental y social del sistema, a través de sus subsistemas (familia, agricultura, silvicultura y ganadería), lo que permitió precisar los elementos cualitativos y cuantitativos de la sustentabilidad. También se utilizó la técnica de entrevista no estructurada que tuvo la finalidad de ahondar más en las respuestas vertidas en los cuestionarios. No se entrevistó a todos los participantes debido a que muchos se rehusaron. Tanto el cuestionario como las guías de entrevista se estructuraron de acuerdo con los elementos de la planificación estratégica. En los reactivos se tuvo cuidado de usar el lenguaje propio de los agricultores. 


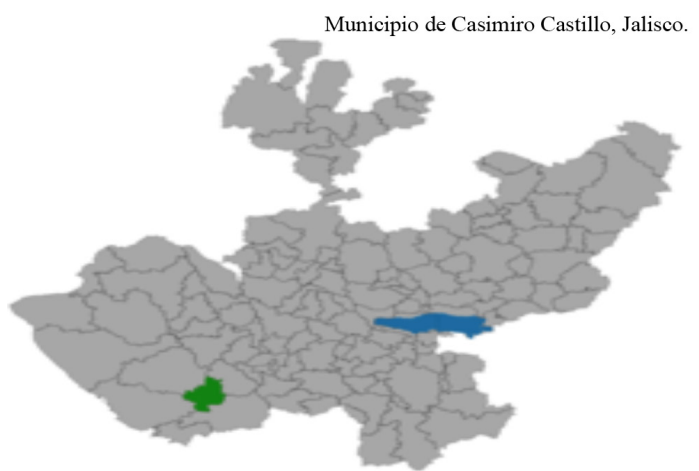

Figura 1. Ubicación geográfica del municipio de Casimiro Castillo, Jalisco, México.

\section{Aplicación de las encuestas}

Todos los participantes fueron informados sobre el propósito de la encuesta y la naturaleza de la investigación. También se les pidió que respondieran el cuestionario voluntariamente y se les informó de la política de confidencialidad de los datos que les habían otorgado.

La encuesta tuvo como objetivo conocer el funcionamiento de la lógica económica, ambiental y social del sistema, a través de sus subsistemas (familiar, agrícola, forestal y ganadero), que permitieron determinar los elementos cualitativos y cuantitativos de la sostenibilidad.

Los ítems del cuestionario se estructuraron de acuerdo con elementos de planificación estratégica. Antes de la aplicación general del cuestionario, se entrevistó a 5 productores, con el fin de verificar la comprensión de las preguntas y la aclaración de dudas y, por lo tanto, eliminar los errores de diseño que pudieran presentarse en el instrumento. Para ello se revisaron los comentarios, preguntas y encuestas a fin de comprobar si todos entendían las preguntas de la misma manera. El propósito de esta prueba fue determinar la capacidad del instrumento $\mathrm{y}$, por lo tanto, verificar que los resultados obtenidos fueran equivalentes entre los encuestados, independientemente de quién lo aplicara.

Con la esta metodología utilizada se obtuvieron los datos necesarios para la determinación de la sustentabilidad, así como la contribución de dichos datos a cada una de las dimensiones (ambiental, social y económica) de la región, municipios y comunidades. Asimismo permitió la identificación cuantitativa y cualitativa de las variables estratégicas centrales para el desarrollo agrícola regional.
La determinación de ISR se complementó con un tercer componente basado en el análisis de la información obtenida de las entrevistas realizadas con 19 productores líderes y representantes de instituciones federales, estatales y municipales.

\section{Sustentabilidad}

El concepto de sostenibilidad utilizado en esta investigación se desarrolla con base en $(f)$ las relaciones que los humanos establecen entre sí (dimensión social), la transformación que hacen de la naturaleza a través de instrumentos y tecnología disponible (dimensión económica) y la capacidad de la naturaleza para desarrollarse y recuperarse de la actividad del hombre (dimensión ambiental). Por lo tanto, la sostenibilidad se calcula en términos de igualdad (Gómez et al., 2017).

Cuantificación y calificación de sostenibilidad

La calificación de cada variable se relacionó con su tendencia hacia la sostenibilidad y la ubicación de los valores promedio reales en un intervalo que se establece en una escala propuesta por el evaluador. Por ejemplo, de $-5 \mathrm{a}+5$ y sus límites están definidos por el intervalo proporcional (Xp), el valor promedio mínimo y el máximo absoluto que la variable toma entre las comunidades (Gómez et al., 2017).

\section{Resultados}

En este estudio se aplicó la misma metodología y estrategia para el análisis de datos que utilizamos en el estudio realizado en 2017. Por lo tanto, de la lista general de las 18 variables de campo fueron seleccionadas por funciones discriminantes las de mayor variación entre las comunidades, reduciendo el número de variables por dimensión a seis para cada dimensión. Las variables presentaron diferencias en los promedios entre las comunidades para la evaluación de la sustentabilidad, que se enumeran por dimensión: Ambiental, Social y Económica en la Tabla 1.

Con las 18 variables se creó una base de datos, a la cual se le aplicó nuevamente el procedimiento de análisis de función discriminante. Así, los siguientes resultados surgen de este análisis.

Con la aplicación de las ecuaciones utilizadas en este estudio, la sustentabilidad del valle agrícola de Casimiro Castillo presentó un IRS $=0,4818$ o 9,74\%, que lo califica como de muy baja sustentabilidad, 
y la dimensión de ausencia de la contribución al desarrollo sustentable (componente ambiental) mostró un IRS $=0,4775 \%$, que se convierte en su limitación. No obstante, en un estudio realizado anteriormente (Gómez et al., 2017), el IRS es casi igual en los municipios del estado de Jalisco, ubicados en la misma región geográfica, Autlán de Navarro $=0,5516 \%$, El Grullo $=0,5363 \%$ y El Limón $=0,5597 \%$.

\section{Discusión}

En el valle hay una dinámica de sustentabilidad muy baja, si se compara el IRS obtenido con el de los municipios de El Limón, El Grullo y Autlán de Navarro en el estudio realizado en 2017. Estos municipios presentaron mayores valores tanto en el aporte como en las dimensiones, lo que contrasta con los valores observados en el IRS de Casimiro Castillo. Esto no significa que el desarrollo para los municipios con los cuales se está comparando a Casimiro Castillo sea bueno, porque según los resultados de dicho estudio, su IRS fue también muy bajo. Esto refleja la necesidad de acciones más efectivas por parte de los gobiernos municipales y de las agencias estatales y federales para los programas de desarrollo que se aplican en la región. Es importante mencionar que la caña de azúcar es el principal cultivo que se realiza en el municipio donde se hizo este estudio y es la razón por la cual se eligió esa actividad como el indicador más representativo para su desarrollo.

\section{Conclusiones}

A partir de los resultados obtenidos en esta investigación, podemos concluir que la sostenibilidad del valle agrícola del municipio de Casimiro Castillo, Jalisco, México, es muy baja, ya que presenta un valor del IRS inferior al encontrado en el estudio realizado en 2017 en los municipios de Autlán de Navarro, El Grullo y El Limón. Ello pone de relieve la necesidad de programas gubernamentales para promover el desarrollo de manera sostenible en beneficio de las comunidades que conforman el municipio.

\section{Literatura Citada}

Bowers, J.

1997. Sustainability and Environmental Economics. An Alternative text. Longman Singapore Publishers Ltd. Singapore. $28 \mathrm{p}$.

Brown, B.J.; Mark E.; Hanson, Siana M.; Liverman, Robert W.; Merideth, Jr.

1987. Global Sustainability: toward definition. Environmental Management, 6: 713-719.

Casas, R.; González, F.; Martínez, T.; García, E.; Peña, B. 2009. Sostenibilidad y Estrategia en Agroecosistemas campesinos de los Valles Centrales de Oaxaca. Agrociencia, 43(3): 319-331.
Gómez, J.E.; Fallad, J.; Hueso E.J.; Castañeda, A. 2017. Three-mensional sustainability profile in the agricultural valley Autlán-El Grullo-El Limón, Jalisco, México. ECORFAN Republic of Paraguay Journal, 3(4): $1-8$.

WCED.

1987. Our Common Future. World Commission on Environment and Development. Oxford University Press. UK. 400 p. 